\title{
Research on the Electrode Materials of Vanadium Redox Flow Battery Jiayue $\mathrm{Yu}^{1, \mathrm{a}}$ \\ ${ }^{1}$ University of California-San Diego, San Diego 92037, United States \\ ajiy048@ucsd.edu
}

Keywords: VRFB, electrodematerial, structure.

\begin{abstract}
The vanadium redox flow batteryisan electrochemical storage system which allows energy to be stored in two solutions containing differentredox couples. Unlike commercially available batteries, all vanadium redox flow batteries have uniqueconfigurations, determined by the size of the electrolyte tanks. This work reviews anddiscusses the progress on electrodes and their reaction mechanisms as key components of thevanadium redox flow battery.
\end{abstract}

\section{Introduction}

Exploration and development of renewable energy sources, likesolar and wind energy, are among the hottest topics of our times.However, to be competitive with coal, natural gas, oil and nuclearpower, the renewable energy needs to be cost effective and reliable.Energy storage, and especially large-scale energy storage, isthe key technology to achieve this. [1] In combination withrenewable resources, energy storage can increase the quality andthe stability of e.g. photovoltaic (PV) and wind-generated electricity.Energy storage can be considered as any technology thatcan convert and store electricity to overcome the mismatchbetween generation and end-use. Also, energy storage can efficientlyincrease the value of electric power by correcting for powerfluctuations in very short time. Fig. 1 clearly shows the differentstorage requirements for electric power utility applications [2].

As one kind of energy storage technique, the vanadium redoxflowbattery (VRFB) is well-suited for large-scale utility applicationsdue to its attractive features such as its long life, active thermalmanagement and independence from energy and power ratings.

\section{The vanadium redox flow battery}

\subsection{The structure of VRFBs}

The VRFB is an electrochemical system that can realize theconversion between chemical energy and electrical energy. Asshown in Fig. 2, VRFBs have two electrolyte tanks containingactive species of vanadium in different valence states. All active species are dissolved ina sulfuric acid medium. The concentrations of vanadium ionsand sulfuric acid are in the range of 1-3 $\mathrm{M}$ and 1-2 $\mathrm{M}$, respectively.During charge-discharge processes, the active species areoxidized or reduced to achieve conversion between chemicalenergy and electrical energy.Two half cells, two solid electrodes and one IEM are the maincomponents of a VRFB single cell. The reactions occur on thesurface of solid electrodes during charge and discharge. Highsurface area carbon materials are normally used as electrodes inVRFB, the materials operate in a certain range of voltage potentialswith minimal hydrogen and oxygen gas evolution. TheIEMs in between isolate both electrolytes, but allow protons tomigrate. 


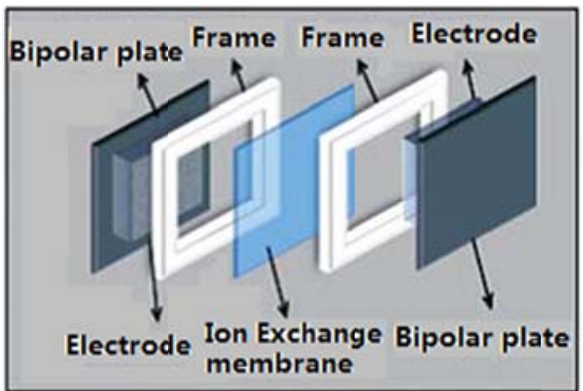

\subsection{The principle of VRFBs}

Fig. 1 The structure of single cell

Featured by the dissolved redoxspecies in the recirculated solution, the flow battery differs fromother batteries storing the energy in electrode structures, such aslead-acid batteries and lithium-ion batteries.VRFBs have a unique advantage over other flowbatteries due to the fact that their positive and negative electrolytesare identical in their discharged sttates. [3] This makes shipmentand storage of electrolyte more simple and inexpensive.Electrical balance is achieved by themigration of proton across a membrane separating the electrolytes.

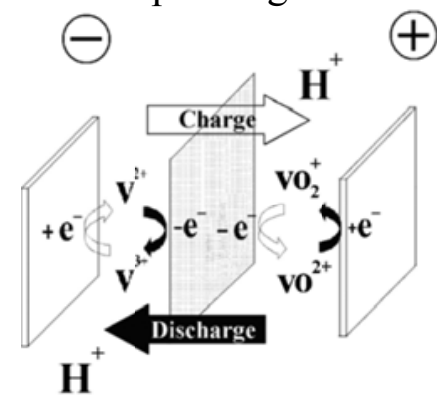

Fig. 2 Schematic illustration of the redox reaction mechanism

The charge-discharge reactions of the VRFB are:

Negative electrode

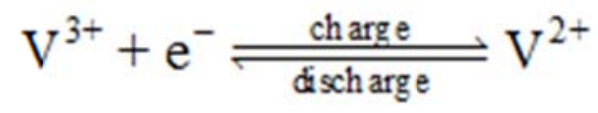

Positive electrode

$$
\mathrm{VO}^{2+}+\mathrm{H}_{2} \mathrm{O} \underset{\text { discharge }}{\stackrel{\text { charge }}{\rightleftharpoons}} \mathrm{VO}_{2}^{+}+2 \mathrm{H}^{+}+\mathrm{e}^{-}
$$

\subsection{The application of VRFBs}

VRFB, as proposed by the Skyllas-Kazacos group fromAustralia in 1984, has received wide attention in energy storagethanks to its long cycle life, flexible design, fast response time,deep-discharge capability, and low pollutant emissionNowadays, the VRFB is becoming more and more mature andfinds extensive applications in different areas, such as renewableenergy, grid management (load leveling and peak shaving), emergency and back-up power stations, electric vehicle chargingstations and communication base stations.

\section{Electrode materials for the VRFB}

An ideal electrode material should provide a high electricalconductivity, good mechanical properties, and strong chemicalresistance, be of reasonable price and have a long cycle life inhighly oxidizing media. Typical electrodes used in RFBs aremade of carbon-based composites or inert metallic materials.Carbon-based electrodes are more common than their metalliccounterparts, as they do not undergo dissolution or formation ofoxide during chemical oxidation. Metal ions from the metallicelectrodes can dissolve into the electrolyte during discharge andcorrosion, which could lead to unstable redox potentials anddisturb the chemistry of the RFBs.[4] If metallic electrodes are usedthey are generally based on noble metals, which have goodelectrochemical stability or catalytic 
property. Some metallicelectrode materials with high overpotentials for gas evolutionhave been used to facilitate the desired reactions and avoid sidereactions.

\subsection{Carbon cloth electrode}

A carbon clothelectrode material was first evaluated for the VRFB by Rychcik and Skyllas-Kazacos in 1987, but a high rate ofmechanical degradation was observed during charging. Thiscan be attributed to the low surface area of carbon cloth materials and the flow-by configuration, whichwould limit diffusion of vanadium ions, leading to increasedpolarization and oxygen co-evolution during charging. Carbon clothwas, however, investigated as anelectrode for the VRFB in 1991 by Kaneko et al, who comparedthe electrochemical properties of GF-20 PAN-type carbon clothand BW-309 cellulose-type carbon cloth electrodes by usingcyclic voltammetry $(\mathrm{CV})$. It was found that the BW-309 cellulose-type carbon cloth electrode showed greater reactivity inboth the anolyte and the catholyte of VRFBs compared with theGF-20 PAN-type carbon cloth electrode.
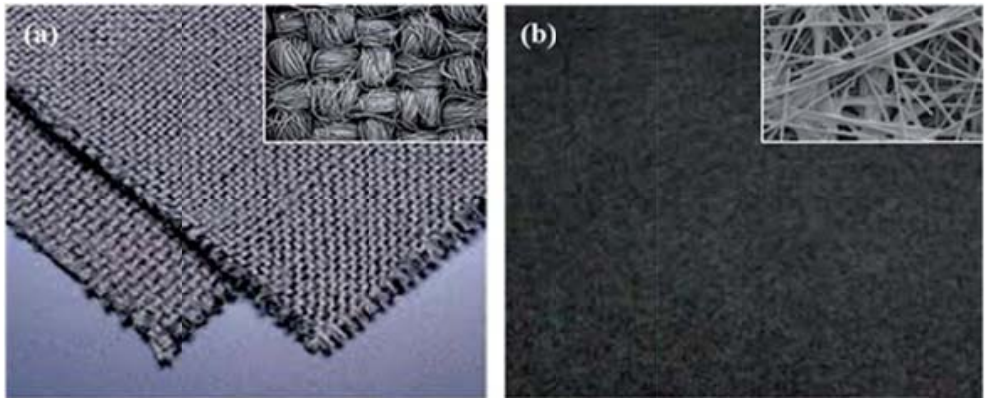

Fig. 3 Material of current commercialized electrodes for the VRFB

(a) carbon cloth electrode, (b) carbon paper electrode

\subsection{Carbon paper electrode}

Carbon paper (Fig. 3b) has promisingproperties such as high porosity, low density, and lowelectrical resistance, which make it a potential candidate for theVRFB. Because of its very low surface area and high activationovervoltage losses compared with carbon felt materials, ithas not been widely used in the VRFB to date. Owing to its otheradvantages, however, efforts to utilize carbon paper as theelectrode for the VRFB were initiated in the late 2000s. The effect of catalyst incorporation into the carbon paperelectrode was also studied by Zhang et al. by coating WO3nanoparticles and activated carbon on the surface.Despite its promising catalytic effect, it could not beused independently because of its low electrical conductivity. Ifused with SAC, however, the electrical conductivity could beeffectively improved, leading to enhanced electrochemicalreactivity for the $\mathrm{V}$ redox reactions in the VRFB.

\subsection{Carbon-polymer composite electrode}

Although not suitablefor use as the electroactive material for the VRFB electrodereactions, carbon-polymer composite electrodes have beendeveloped for use as the VRFB electrode substrate.In fact, the electrochemicalproperties of the electrode are highly dependent onthe choice of binder materials, as well as the composition,mechanical properties, and electrical conductivity of the electrode.In practice, by applying the optimized carbon-polymercomposite electrode in VRFBs, a high voltage efficiency of $91 \%$ could be attained. [5] A few investigations on composite electrodeswere conducted in the initial stages of the developmentof the VRFB. Haddadi-Als et al. reported the electrochemical stability and degradation behavior of a carbon-polyolefin composite electrode, and Huang et al. reported good reversibilityand reactivity towards the V redox reactions of acomposite electrode composed of carbon black, graphitepowder, and a binder. The best performance was obtainedwhen the ratio of graphite powder to carbon black was 3: 1.According to Wang et al., porous graphite composite electrodescoated with Co showed a high voltage efficiency of $81.5 \%$,thanks to lower charge transfer resistance.

\subsection{PAN-type carbon felt or graphite felt electrode.}

Ever since theinitial development of the VRFB, PAN-based carbon or graphitefelt electrode has been the most widely used electrode material for theVRFB.The PAN-type carbon felt electrode is known to have alarge specific surface area and high electrical conductivity, as well as excellent 
stability in both chemical and electrochemicalenvironments. As with other types of carbon and graphitematerials, however, the low hydrophilicity of the PAN-typecarbon felt electrode requires improvement to enhance itsreactivity towards the $\mathrm{V}$ redox reactions in aqueous solution.Thermal treatment of carbon materials - by heating theirsurface - is a traditional approach developed 100 years ago,and even now, it is the most common and easy way to integrateabundant oxygen functional groups onto the surface of thecarbon materials.

\subsection{Graphene or graphene oxide (GO) electrode}

Graphene, atwo-dimensional graphitic material with sp2-hydridized carbon atoms arranged in a hexagonal lattice, has a large specific surface area and excellent electrical conductivity, and thanks toits intrinsic properties, it can be utilized in a variety of fields. Compared toa graphite electrode, the graphite-graphene composite electrode with graphene as filler showed noticeable reactivity in the VRFB catholyte.Graphene oxide nanoplatelet (GONP) materials have alsobeen investigated as electrodes for the VRFB. The GONP electrodeoxidized at $50{ }^{\circ} \mathrm{C}$ was found to give the best performance in boththe anolyte and the catholyte of the VRFB. The electrochemicalenhancement of GONP is mainly attributed to the large amountof $\mathrm{C}-\mathrm{OH}$ and $\mathrm{COOH}$ functional groups formed during theoxidation process. Despite the excellent electrochemical reactivityof GONP, however, it has the shortcoming of comparativelylow rate capability due to its low electrical conductivity.

\section{Summary}

A detailed historical review is presented here to elucidate thetechnical trends in electrode materials research for the VRFB.To further enhance the electrochemical properties ofcarbon based electrodes various surface treatments wereproposed. The early studies focused on attaching various oxygen functionalgroups to the surfaces of the electrodes. Later on, certainOH-functional groups were widely accepted as providing activereaction sites for the vanadium redox couple reactions, enhancing the reactivity and wettability of the carbon electrodes.Carbon and graphite will continue to form the basis of theelectrode material for the VRFB, but future research will focuson the use of thin carbon cloth or paper as part of "zero-gap"cell architecture for a high-power-density VRFB stack.

\section{References}

[1] Qian Xu, Yonglian Qiao: Chinese Journal of Power Sources, Vol. 32 (2008) No.12, p.823.

[2] Wang Jiamin, Hu Junping, Liu Chunhua, Shi Siqi: Lithium ion battery storage project, Vol. 41 (2012) No. 2, p.95.

[3] Leipeng Zhang, Wei Wang: Chinese Journal of Power Sources, Vol. 36 (2014) No.6, p.1176.

[4] Zelang Jian, Novel Electrode Materials for StationaryBatteries(Ph.D., Wuhan University of Technology, China 2012), p.67.

[5] Information on http://baike.baidu.com/view/1949936.htm 